\title{
Variabilidade diária da temperatura do solo: Um estudo de caso
}

\author{
Daily variability of soil temperature: A case study
}

\author{
Júlio Mannuel Tavares Diniz ${ }^{1}$, Edicarlos Pereira de Sousa ${ }^{2}$, José Alberto Calado Wanderley ${ }^{3}$, José Fideles Filho ${ }^{4}$, \\ Patrício Borges Maracajá ${ }^{5}$
}

\begin{abstract}
Resumo: Mesmo sabendo-se que a temperatura do solo é um dos fatores ambientais de maior importância para a agricultura, poucas pesquisas foram realizadas até então a respeito dessa variável. Logo, o presente trabalho tem como objetivo o estudo das características térmicas do solo através de dados coletados no Instituto Nacional do Semiárido (INSA), durante os meses de julho a dezembro do ano de 2012. Para avaliar a magnitude da difusividade térmica do solo foram utilizados os métodos da amplitude, do arco tangente e o logarítmico. A partir da análise dos resultados verificou-se boa consistência das estimativas da difusividade térmica do solo obtidas pelos métodos distintos.
\end{abstract}

Palavras-chave: INSA, características térmicas, difusividade térmica, agricultura.

Abstract: Even knowing that soil temperature is an environmental factor of great importance to agriculture, few researches were performed until then about this variable. Therefore, this work aims the study of thermal characteristics of the soil using data collected in National Institute of Semiarid (INSA), during the months from july to december of 2012. To evaluate the magnitude of the soil thermal diffusivity were used methods of amplitude, of the arctangent and the logarithmic. From the analysis of the results it was found good consistency of the estimates of soil thermal diffusivity obtained by different methods.

Keywords: INSA, thermal characteristics, thermal diffusivity, agriculture.

\section{INTRODUÇÃO}

As condições de temperatura do solo estão em constantes mudanças visto que este está sendo continuamente perturbado pela "entrada" de calor mediante incidência de radiação solar como também pela "saída" através de processos como a evaporação (LAL \& SHUKLA, 2004). Informações acerca de temperatura do solo, sua magnitude e a sua forma de variação no tempo e no espaço são elementos de importância primordial para a determinação da taxa e direção dos processos físicos existentes no solo (HILLEL, 2004).

Medições de temperatura em diferentes profundidades do solo são rotineiras em estações meteorológicas, porém, são poucos os estudos que têm feito uso de tais observações. Em geral, a não utilização de tal acervo de dados se deve principalmente por não considerar o fator térmico como limitante na produção agrícola, dando-se maior ênfase ao fator hídrico.

Para o crescimento satisfatório das plantas é essencial que o solo propicie um ambiente com características físicas favoráveis para o desenvolvimento de suas raízes, a fim de que estas se tornem capazes de explorar o solo suficientemente de modo que atenda as necessidades de água, nutrientes e ancoragem das plantas (WARRICK, 2001).
A temperatura do solo é uma propriedade de natureza física que influi diretamente em uma série de processos ambientais relacionados às plantas tais como germinação de sementes, velocidade e duração de crescimento, desenvolvimento e atividade radicular, ocorrência e severidade de pragas, etc. (BRADY, 1989).

$\mathrm{O}$ aquecimento demasiado do solo na fase inicial de estabelecimento das culturas compromete a absorção de nutrientes pelas plantas (CASTRO, 1989). As altas temperaturas também ocasionam efeitos nocivos sobre as raízes e a atividade microbiana (FURLANI et al., 2008). Johnson \& Lowery (1985) verificaram que a variação de $1^{\circ} \mathrm{C}$ na temperatura do solo pode afetar significativamente a taxa de crescimento do milho em regiões de clima temperado.

Quando uma mesma quantidade de energia está disponível para solos distintos, o processo de aquecimento e resfriamento pode ser bastante variável em virtude de suas propriedades térmicas específicas, dentre as quais se pode destacar a difusividade térmica (MOTA, 1983). Esta, por sua vez, é um indicativo da capacidade do solo em transportar calor através de seu perfil vertical, ou seja, reflete a facilidade com que são modificadas as temperaturas.

Recebido em 30122012 e aceito em 28032013

${ }^{1}$ Graduado em Física (UEPB), Mestrando em Meteorologia UFCG, Av. Aprígio Veloso 882, Bodocongó, CEP 58109-970, Campina Grande, PB. . Email: julio_mannuel@hotmail.com

${ }^{2}$ Graduado em Matemática (UFCG), Doutorando em em Meteorologia UFCG, Av. Aprígio Veloso 882, Bodocongó, CEP 58109-970, Campina Grande, PB. . Email: edicarlos.p.sousa@gmail.com

${ }^{3}$ Eng $^{\circ}$ Agronomo, Doutorando em Engenharia Agrícola, UAEA/UFCG, Av. Aprígio Veloso 882, Bodocongó, CEP 58109-970, Campina Grande, PB. Email: alberto_agronomo@hotmail.com

${ }^{4}$ Doutor em Recursos Naturais, Professor UEPB

${ }^{5}$ Prof D. Sc. Da UFCG CCTA Email patricio@ufcg.edu.br Revista Verde (Mossoró - RN - Brasil), v. 8, n. 1, p. 01 - 06, jan/mar de 2013 (Nota Tecnica Cientifica) 
Em virtude de sua importância e pelo fato de cada solo possuir propriedades e características térmicas específicas, torna-se interessante o estudo do comportamento térmico do solo para cada situação particular. Até então, poucos estudos dessa natureza foram realizados pela comunidade científica para os solos brasileiros.

A fim de contribuir para o desenvolvimento dessa área científica, o presente trabalho tem como objetivo o estudo do comportamento diário e propriedades térmicas do solo (particularmente a difusividade térmica) a partir de dados de temperatura obtidos na cidade de Campina Grande-PB.

\section{MATERIAL E MÉTODOS}

Para o desenvolvimento do presente trabalho foram utilizados dados obtidos através de uma estação meteorológica automática em funcionamento no Instituto Nacional do Semiárido (INSA), localizada na cidade paraibana de Campina Grande $(7,22 \mathrm{oS} ; 35,88 \mathrm{oW})$. Os dados analisados são provenientes dos meses de julho a dezembro do ano de 2012.

Para o monitoramento térmico do solo foram utilizados sensores de temperatura que operam durante 24 horas por dia e encontram-se instalados em três profundidades distintas do solo. Esses equipamentos estão

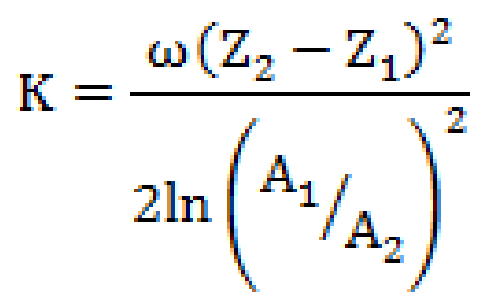

$\mathrm{Na}$ equação acima, $\omega$ é a frequência angular $(7,27 \times 10$ $\left.{ }^{5} \mathrm{rad} / \mathrm{s}\right), \mathrm{A}_{1}$ e $\mathrm{A}_{2}$ são as amplitudes da onda de temperatura nas profundidades $Z_{1}$ e $Z_{2}$ (diferença entre a temperatura às profundidades de 10,20 e $50 \mathrm{~cm}$. A fim de estimar a difusividade térmica considerou-se o solo um meio homogêneo, isotrópico, sem fontes ou sumidouros de calor, restringindo-se a análise apenas dos processos que ocorrem na direção vertical. A partir dessas considerações irá ser aplicado o tratamento clássico de condução de calor a um meio homogêneo proposto por Fourier, que permite conhecer as amplitudes e fases da onda de calor (FIDELES FILHO, 1988).

Uma vez que a difusividade térmica do solo é calculada para certas camadas específicas do solo, faz-se necessário que sejam destacados os critérios adotados nesse estudo. Devido à disponibilidade de dados, as estimativas foram realizadas para as porções do solo denominadas de camada 1 (estende-se desde 0,1 a $0,2 \mathrm{~m}$ de profundidade), camada 2 (estende-se desde 0,2 a $0,5 \mathrm{~m}$ de profundidade) e camada 3 (estende-se desde 0,1 a 0,5 $\mathrm{m}$ de profundidade).

Mediante as informações e considerações anteriores, os métodos da amplitude, do arco tangente e o logarítmico foram empregados com o intuito de se estimar a magnitude dessa grandeza física cujos detalhes podem ser encontrados em Gao et al. (2009).

Para se estimar a difusividade térmica do solo através do método da amplitude foi utilizada a seguinte equação:

$$
\mathrm{K}=\frac{\omega\left(\mathrm{Z}_{2}-\mathrm{Z}_{1}\right)^{2}}{2\left\{\arctan \left[\frac{\left(\mathrm{T}_{1}-\mathrm{T}_{3}\right)\left(\mathrm{T}_{2}^{s}-\mathrm{T}_{4}^{s}\right)-\left(\mathrm{T}_{2}-\mathrm{T}_{4}\right)\left(\mathrm{T}_{1}^{s}-\mathrm{T}_{3}^{\circ}\right)}{\left(\mathrm{T}_{1}-\mathrm{T}_{3}\right)\left(\mathrm{T}_{1}^{s}-\mathrm{T}_{3}^{s}\right)+\left(\mathrm{T}_{2}-\mathrm{T}_{4}\right)\left(\mathrm{T}_{2}^{s}-\mathrm{T}_{4}^{\circ}\right)}\right]\right\}^{2}}
$$

máxima do dia e a média diária), respectivamente fazendo uso do método do arco tangente para o cálculo da difusividade térmica, aplicou-se a expressão abaixo:

Onde:

$\mathrm{T}_{1}, \mathrm{~T}_{2}, \mathrm{~T}_{3}$ e $\mathrm{T}_{4}$ são as temperaturas obtidas na profundidade;

$\mathrm{Z}_{1}$ e T' ${ }_{1}, \mathrm{~T}_{2}, \mathrm{~T}_{3}{ }_{3}$ e $\mathrm{T}{ }_{4}$ são as temperaturas oriundas no nível $\mathrm{Z}_{2}$.

Em ambas as profundidades, foram utilizadas temperaturas observadas em intervalos de 6 em 6 horas.

O método logarítmico é análogo ao do arco tangente, principalmente no que diz respeito ao intervalo tempo (6 horas) entre as temperaturas utilizadas para se estimar a difusividade térmica. Nesse método, o calculo é realizado através da relação

\section{RESULTADOS E DISCUSSÃO}

A partir dos dados de temperatura do solo, coletados em três profundidades distintas nos meses de julho a dezembro do ano de 2012 para a estação meteorológica automática do INSA, foram calculadas as 
médias horárias de temperatura tendo em vista que esses valores, como expostos na Figura 1, representam o ciclo térmico diário médio do solo para o respectivo período.

as variações diárias de temperatura não são tão acentuadas como nas demais regiões do globo terrestre.
Esse procedimento se justifica devido à localização da área de estudo (próxima ao equador geográfico) e por isso

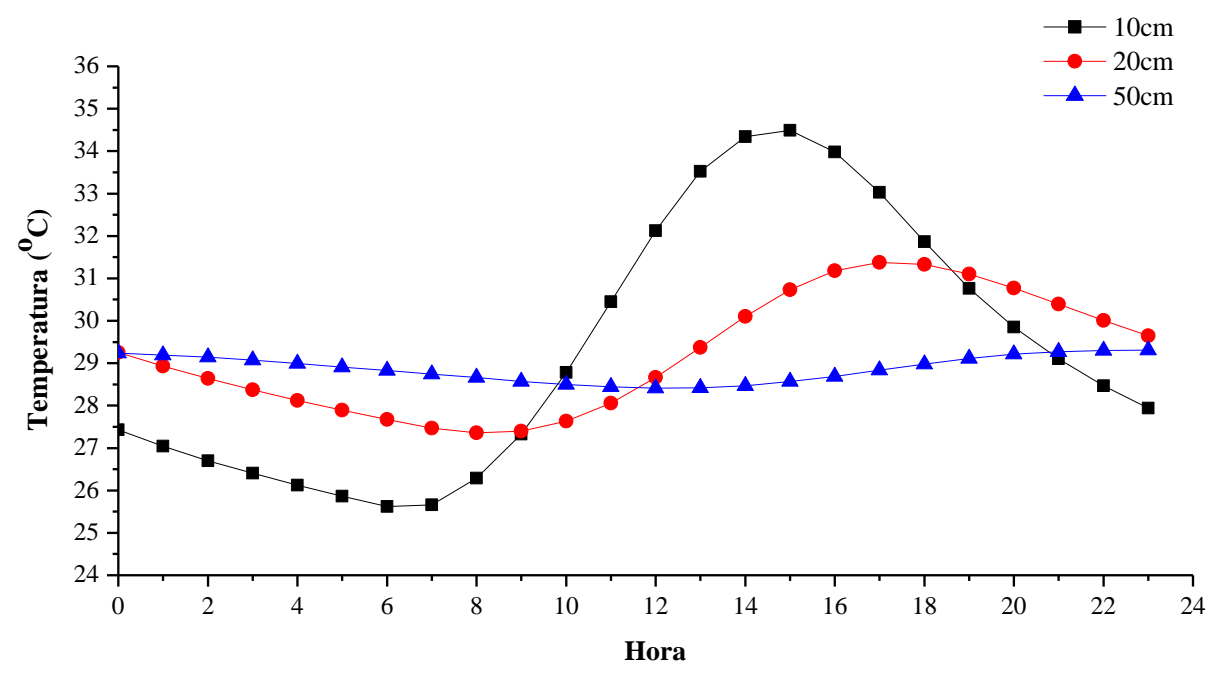

Figura 1. Comportamento médio diário das temperaturas do solo em diferentes profundidades.

Mediante a análise da figura acima, se verifica que até por volta das 10 horas da manhã há um progressivo aumento das temperaturas à medida que se avança em profundidade com os valores máximos sendo observados em $50 \mathrm{~cm}$. Após esse período, em consequência da incidência mais acentuada de radiação solar, a temperatura da superfície do solo e regiões adjacentes passa a apresentar um significativo aumento em magnitude com valores máximos sendo observados em torno das 15 horas. O decréscimo das temperaturas observadas em seguida é consequência do decaimento da disponibilidade energética.

Verifica-se também na Figura 1 que as temperaturas do solo permanecem quase que constante durante o ciclo diário na profundidade de $50 \mathrm{~cm}$. As variações acentuadas observadas nas porções mais próximas a superfície estão associadas ao fato de sua maior facilidade em ganhar e perder calor durante o ciclo diário. Gasparim et al. (2005) observou fenômeno semelhante ao estudar o comportamento térmico do solo em um experimento realizado e conduzido na Estação Experimental Agrometeorológica da Universidade Estadual do Oeste do Paraná.

As variações diárias de temperatura do solo estão diretamente relacionadas à disponibilidade energética e inversamente relacionadas à profundidade. Entretanto, os fenômenos meteorológicos que ocorrem sob a superfície do solo afetam diretamente o seu regime de temperatura. A precipitação e a nebulosidade diária são exemplos de elementos que, muitas vezes, fazem os ciclos térmicos diários diferirem significativamente do exposto na Figura 1. Na ausência destes, as diferenças estão basicamente associadas às magnitudes das temperaturas.

A análise agora irá se restringir ao comportamento térmico do solo para horas específicas do ciclo diário. A Figura 2 apresenta as variações diárias da temperatura para o perfil vertical do solo observadas nos horários de $6 \mathrm{~h}(\mathrm{~A}), 12 \mathrm{~h}(\mathrm{~B}), 18 \mathrm{~h}(\mathrm{C})$ e $24 \mathrm{~h}$ (D) UTC, ou seja, 3h, 9h, 15h e $21 \mathrm{~h}$ (horário local). 

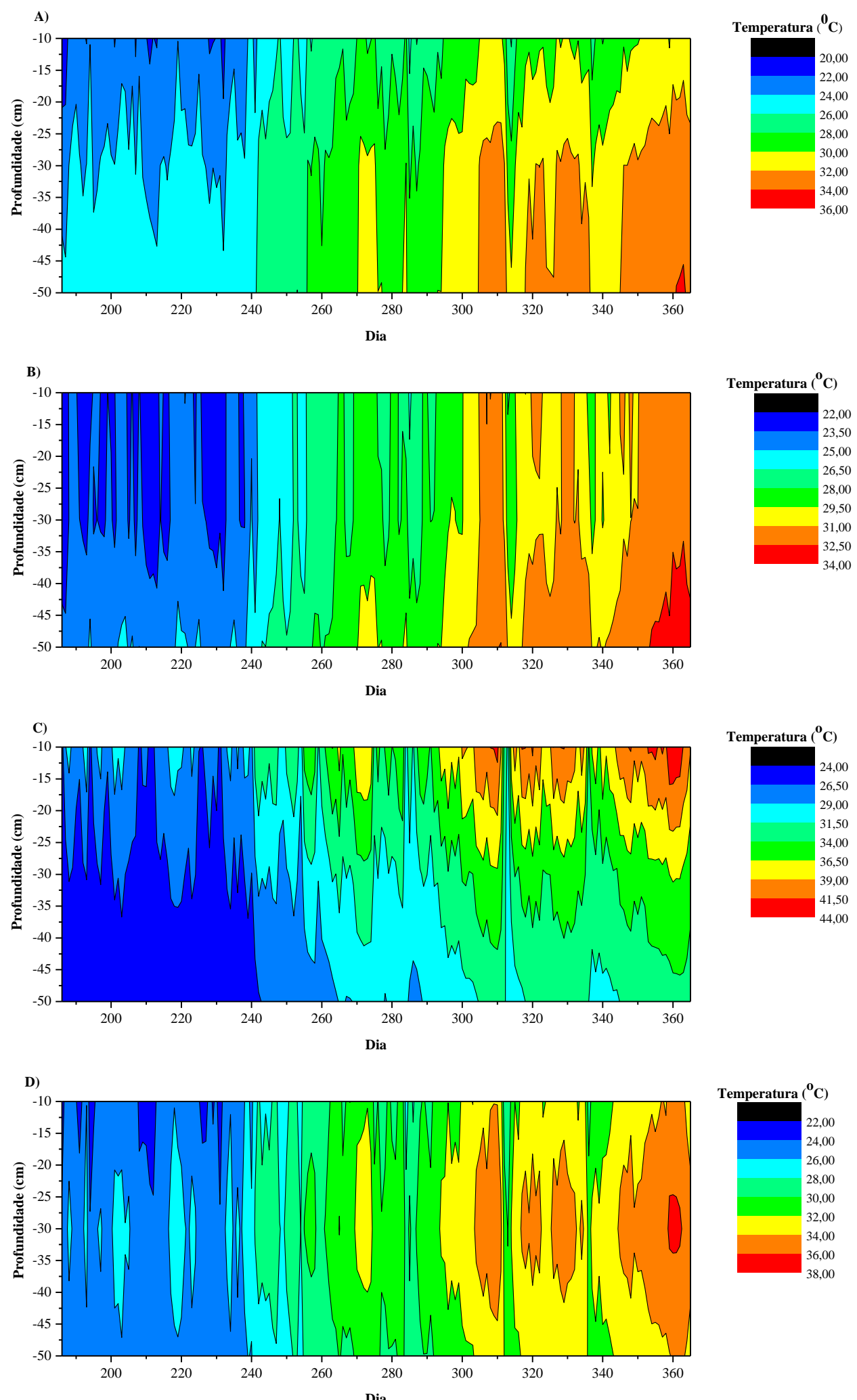

Figura 2. Variabilidade diária do perfil vertical de temperatura do solo às 6 h $(A), 12 h(B), 18 h(C)$ e $24 h(D)$ UTC. 
Observando a Figura 2 é possível verificar o aumento gradual da temperatura do solo, com os máximos valores sendo alcançados no mês de dezembro. Esse comportamento era esperado em virtude das observações se iniciarem no inverno (pouca disponibilidade energética) e terminarem no verão (elevada disponibilidade energética). Portanto, pode-se observar que as maiores temperaturas são observadas de dezembro a março, enquanto que os menores valores dessa variável se encontram entre os meses de junho e setembro. A amplitude térmica anual das temperaturas do solo não é muito acentuada se comparada a localidades situadas em médias e altas latitudes. Isso mostra que não há durante o ano variações significativas das condições meteorológicas em regiões localizadas próximas ao equador terrestre.

Características distintas podem ser observadas ao analisar separadamente cada caso exposto na figura acima. Na Figura 2A é nítido o aumento gradual da temperatura à medida que se avança em profundidade, com os mínimos valores sendo verificados próximo da superfície, reflexo da acentuada facilidade (dificuldade) com que as porções mais superficiais (profundas) têm em perder (ganhar) calor durante o ciclo diário. Apesar da incidência de radiação solar, observa-se que esta não é ainda suficiente para aquecer a superfície nas primeiras horas do dia, de modo que, conforme mostra a Figura 2B, as temperaturas mais elevadas permanecem sendo verificadas nas regiões mais profundas do solo. A inversão do gradiente térmico, como se verifica na Figura $2 \mathrm{C}$, é proveniente da significativa incidência de radiação solar, passando as porções mais superficiais (profundas) do solo a possuírem as temperaturas de maior (menor) magnitude. Valores de temperatura elevados observados em profundidades intermediárias, visto na Figura 2D, advêm do fato de não ter transcorrido o tempo necessário para que a camada tenha uma perda de calor suficiente para ocorrer o decaimento significativo de sua temperatura.

Os dados de temperatura foram utilizados para estimativa da difusividade térmica média do solo para o período estudado. Na Tabela 1 são apresentados esses valores calculados para três camadas distintas do solo mediante o uso dos métodos da amplitude, do arco tangente e o logarítmico.

Tabela 1. Difusividade térmica do solo $\left(\mathrm{m}^{2} / \mathrm{s}\right)$ para o período compreendido entre julho e dezembro do ano de 2012 .

\begin{tabular}{cccc}
\hline Método & Camada 1 & Camada 2 & Camada 3 \\
& & & $1,16 \mathrm{E}-06$ \\
Amplitude & $0,63 \mathrm{E}-06$ & $1,50 \mathrm{E}-06$ & $4,58 \mathrm{E}-06$ \\
Arco tangente & $0,68 \mathrm{E}-06$ & $1,98 \mathrm{E}-06$ & $1,13 \mathrm{E}-06$ \\
Logarítmico & $0,54 \mathrm{E}-06$ & $1,55 \mathrm{E}-06$ & \\
\hline
\end{tabular}

Observa-se na Tabela 1 que os métodos se adequam a estimativa dessa variável, visto que os valores concordam significativamente entre si. Algumas discrepâncias surgem na camada 3 ao comparar os valores oriundos do método do arco tangente com os demais. Essas divergências não comprometem o método, porém, sugerem que este seja aplicado em outras situações a fim de verificar sua confiabilidade.

Pela análise da Tabela 1, constata-se também que as maiores magnitudes da difusividade térmica do solo estão associadas à camada $2(0,2-0,5 \mathrm{~m}$ de profundidade). Logo, conclui-se que essa região do solo possui maior capacidade de transferir calor através de seu perfil vertical. Essa característica pode estar associada ao maior teor de água nessa região particular do solo.

\section{CONCLUSÕES}

A partir da análise dos resultados, verificou-se que as variações diurnas e sazonais na incidência de radiação solar fazem com que as condições de temperatura do solo estejam em constante alteração. As porções mais próximas à superfície são as que apresentam maior variação de temperatura durante os ciclos médios diários. Em contrapartida, à medida que se avança em profundidade, as variações médias diárias da temperatura do solo tendem a se tornar cada vez mais discretas.

Os diferentes métodos utilizados para a estimativa da difusividade térmica diária do solo forneceram em geral valores bastante semelhantes, indicando a consistência das metodologias. Estudos semelhantes a este são sugeridos, sendo de fundamental importância que sejam realizados em localidades para as quais não se tenha nenhum conhecimento sistematizado acerca das características térmicas do solo.

\section{AGRADECIMENTOS}

Os autores agradecem a AESA (Agência Executiva de Gestão das Águas do Estado da Paraíba) pelo fornecimento dos dados utilizados nesse estudo, bem como ao Conselho Nacional de Desenvolvimento Científico e Tecnológico (CNPq) e a Coordenação de Apoio ao Pessoal de Nível Superior (CAPES) pela concessão de bolsas de estudo. 


\section{REFERÊNCIAS BIBLIOGRÁFICAS}

BRADY, N. C. Natureza e propriedades do solo. $2^{\text {a }}$ Ed. Rio de Janeiro: Freitas Bastos, 1989;

CASTRO, O. M. Preparo do solo para a cultura do milho. Campinas: Fundação Cargill, 1989;

FIDELES FILHO, J. Estrutura térmica de solos do Nordeste do Brasil. 1988. 85 f. Dissertação (Mestrado em Meteorologia) - Universidade Federal da Paraíba, Campina Grande, 1988;

FURLANI, C. E. A.; GAMERO, C. A.; LEVIEN, R.; Silva, R. P.; Cortez, J. W. Temperatura do solo em função do preparo do solo e do manejo da cobertura de inverno. Revista Brasileira de Ciência do Solo, v. 32, p. 375-380, 2008;

GAO, Z.; WANG, L.; HORTON, R. Comparison of six algorithms to determine the soil thermal diffusivity at a site in the Loess Plateau of China. Hydrology and Earth System Sciences Discussions, v. 6, p. 2247-2274, 2009;

GASPARIM, E.; RICIERI, R. P.; SILVA, S. L.; DALlACORT, R.; GNOATTO, E. Temperatura no perfil do solo utilizando duas densidades de cobertura e solo nu. Acta Scientiarum, v. 27, p. 107-115, 2005;

HILLEL, D. Introduction to environmental soil physics. Massachusetts: Elsevier Science, 2004;

JOHNSON, M. D.; LOWERY, B. Effect of three conservation tillage practices on soil temperature and thermal properties. Soil Science Society of America Journal, v. 49, p. 1547-1552, 1985;

LAL, R.; SHUKLA, M. K. Principles of soil physics. New York: Marcel Dekker, 2004;

MOTA, F. S. Meteorologia agrícola. São Paulo: Nobel S/A, 1983;

WARRICK, A. W. Soil physics companion. Florida: CRC Press, 2001. 